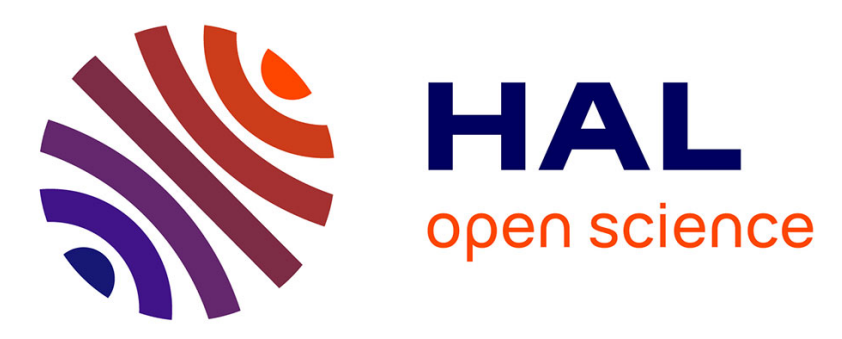

\title{
Accuracy and kinematics consistency of marker-based scaling approaches on a lower limb model: a comparative study with imagery data
}

Pierre Puchaud, Christophe Sauret, Antoine Muller, Nicolas Bideau, Georges Dumont, Hélène Pillet, Charles Pontonnier

\section{To cite this version:}

Pierre Puchaud, Christophe Sauret, Antoine Muller, Nicolas Bideau, Georges Dumont, et al.. Accuracy and kinematics consistency of marker-based scaling approaches on a lower limb model: a comparative study with imagery data. Computer Methods in Biomechanics and Biomedical Engineering, 2019, 23 (3), pp.1-12. 10.1080/10255842.2019.1705798 . hal-02546021

\section{HAL Id: hal-02546021 \\ https://hal.science/hal-02546021}

Submitted on 17 Apr 2020

HAL is a multi-disciplinary open access archive for the deposit and dissemination of scientific research documents, whether they are published or not. The documents may come from teaching and research institutions in France or abroad, or from public or private research centers.
L'archive ouverte pluridisciplinaire HAL, est destinée au dépôt et à la diffusion de documents scientifiques de niveau recherche, publiés ou non, émanant des établissements d'enseignement et de recherche français ou étrangers, des laboratoires publics ou privés. 


\title{
Accuracy and kinematics consistency of marker-based scaling approaches on a lower limb model: a comparative study with imagery data
}

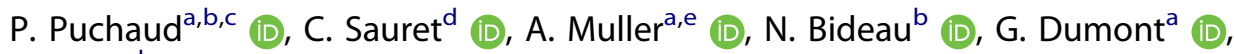 \\ H. Pillet ${ }^{d}$ (ID) and C. Pontonnier ${ }^{a, c}$ (ID

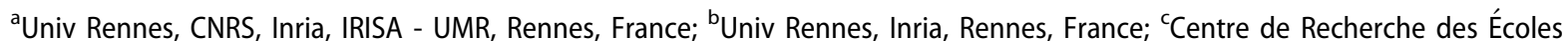 \\ de St-Cyr Coëtquidan (CREC), Guer, France; ${ }^{d}$ Institut de Biomécanique Humaine Georges Charpak, Arts et Métiers ParisTech, France; \\ 'Institut de Recherche Robert-Sauvé en Santé et en Sécurité du Travail (IRSST), Montréal, QC, Canada
}

\begin{abstract}
Medical images are not typically included in protocol of motion laboratories. Thus, accurate scaling of musculoskeletal models from optoelectronic data are important for any biomechanical analysis. The aim of the current study was to identify a scaling method based on optoelectronic data, inspired from literature, which could offer the best trade-off between accurate geometrical parameters (segment lengths, orientation of joint axes, marker coordinates) and consistent inverse kinematics outputs (kinematic error, joint angles). The methods were applied on 26 subjects and assessed with medical imagery building EOS-based models, considered as a reference. The main contribution of this paper is to show that the marker-based scaling followed by an optimisation of orientation joint axes and markers local coordinates, gives the most consistent scaling and joint angles with EOS-based models. Thus, when a non-invasive mean with an optoelectronic system is considered, a marker-based scaling is preliminary needed to get accurate segment lengths and to optimise joint axes and marker local coordinates to reduce kinematic errors.

Abbrevations: AJC: Ankle joint centre; CKE: cumulative kinematic error; DoF: degree of freedom; EB: EOS-based; HB: height-based; HJC: hip joint centre; KJC: knee joint centre; MB: marker-based; MSM: musculoskeletal models; SPM: statistical parametric mapping; STA: soft tissue artifact; $\mathrm{EB}_{a . m^{*}}$ : EOS-based with optimised joint axes, and all model markers coordinates; $\mathrm{MB}_{a . m^{*}}$ : markerbased with optimised joint axes, and all model markers coordinates; $\mathrm{MB}_{\text {l.a.m: }}$ : marker-based with optimised segment lengths, joint axes, and selected model markers coordinates; ASIS: anterior superior illiac spine; PSIS: posterior superior illiac spine
\end{abstract}

\author{
KEYWORDS \\ Scaling; motion capture; \\ EOS; non-invasive; \\ numerical kinematics; \\ optimisation
}

\section{Introduction}

Accuracy of musculoskeletal analyses relies on multiple factors, in which the scaling of the musculoskeletal model (MSM) to the subject is of primary importance. The scaling is generally performed at multiple layers: muscle, inertial, and geometrical. The latter is fundamental to compute accurate joint angles and geometrical parameters (segment lengths, orientations of joint axes, and anatomical positions corresponding to marker local coordinates or muscle insertions) that are necessary to compute muscle paths and moment arms (van den Bogert et al. 2013). Therefore, a scaling method should provide accurate geometrical parameters to ensure a non-negative impact on the inverse kinematics outputs.

Models based on medical images are the current gold standard, they provide the most accurate geometrical parameters (Scheys et al. 2006; Blemker et al. 2007; Valente et al. 2014). In such methods, data from MRI (Kainz et al. 2016, 2017; Halonen et al. 2017), $\operatorname{EOS}^{\circledR}$ (Clément et al. 2015), or CT-scans (Bartels et al. 2015; Marra et al. 2015) was used to reconstruct 3D bone geometries through image segmentation manually (Valente et al. 2014) or semi-automatically (Scheys et al. 2005). Generally, imagery data acquisition and post-processing is time-consuming. It limits its use to small cohorts (Handsfield et al. 2014) and prevents any routine protocol. More recently, several authors proposed to use anthropometric similarities to find the closest model within a database of models extracted from MRIs and scaling it proportionally to the subject (Ding et al. 2019; Klemt et al. 2019).

Scaling methods based on optoelectronic data are used in many studies (Hamner and Delp, 2013; 


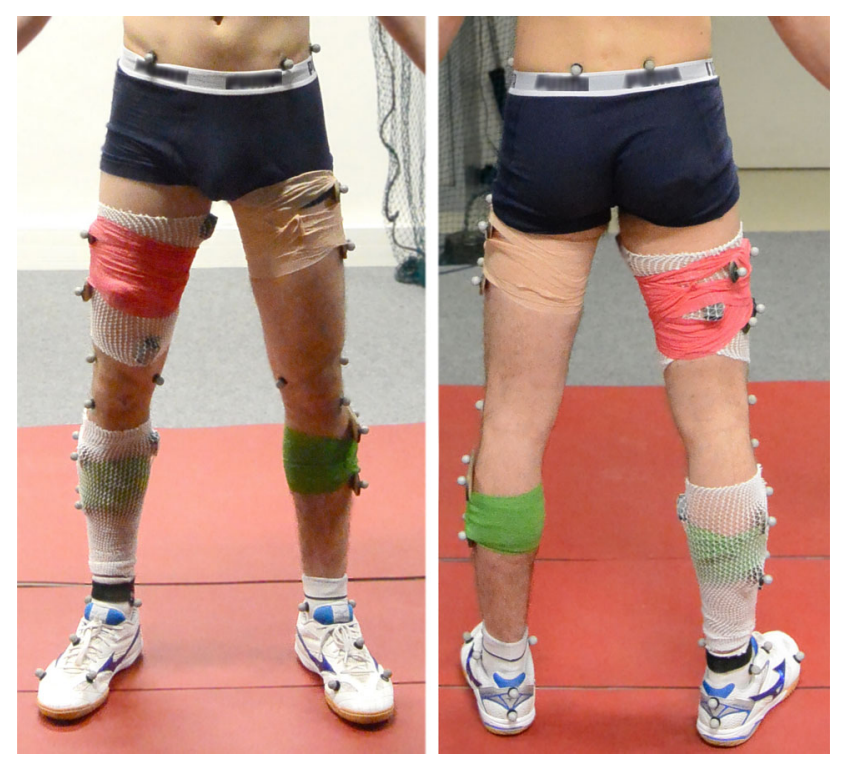

Figure 1. Subject equipped with 30 reflective markers (14 single markers and 16 markers on technical clusters placed on both thighs and shanks). A technical marker was placed on the left iliac crest only for labelling purpose. Foot markers were not included in this study. Markers were placed by skilled experimenters who received a training in palpatory anatomy.

Dupré et al. 2018; Muller et al. 2019a) since they require less required time, knowledge and money.

Many computational methods were developed, including linear scaling (proportional scaling of the segment lengths based on the marker placements, Rassmussen et al. 2005), non-linear scaling (e.g., morphing, radial-basis functions, ..., Lund et al. 2015; Zhang et al. 2016; Nolte et al. 2019 ), and optimisation-based scaling (van den Bogert et al. 1994; Reinbolt et al. 2005, 2007; Andersen et al. 2010b; Lund et al. 2015; Muller et al. 2015a) The optimisation-based scaling relies on dynamic trials according to Lund et al. 2015 and consists in minimising over a selected time interval the least-squares error between experimental markers and their positions on the predefined kinematic model while adjusting geometrical parameters. This approach tends to distribute the experimental (soft tissue artefacts (STA), marker errors, ...) and model errors on the optimised geometrical parameters. Softwares such as OpenSim (Delp et al. 2007), AnyBody (Damsgaard et al. 2006) and CusToM (Muller et al. 2019b) use this geometrical scaling method. This optimisation ensures a low kinematic error (mean distance between experimental and model markers over all the frames of a given trial) after running inverse kinematics. However, the optimised geometrical parameters cannot be directly validated without medical images.
A few studies proposed direct validations of segment lengths scaled from optoelectronic data with medical images. For example, in Kainz et al. (2017) and Bartels et al. (2015), the Delp leg model (Delp et al. 1990) was linearly scaled with the Opensim software and segment lengths were compared to CTscans and MRI extractions. The studies highlighted differences between linear scaling and medical imagery up to respectively 30 and $100 \mathrm{~mm}$ for the femur length. However, these studies only relied on one scaling approach and they did not investigate the geometrical scaling effects on joint angles.

Therefore, the aim of the current study was to identify a scaling method based on optoelectronic data offering the best trade-off between accurate geometrical parameters (segment lengths, orientation of joint axes, marker coordinates) and consistent inverse kinematics outputs (joint angles and kinematic error). For this purpose, 5 scaling methods were applied to a lower limb model on 26 subjects: two image-based scaling methods, one linear scaling methods, and two optimisation-based scaling methods. Kinematic errors and joint angles were evaluated on hip- and kneejoint functional movements and gait. The primary assumption of the study was that optimisation-based scaling methods should provide the best trade-off between geometrical parameters accuracy with respect to image-based methods and ensure the best inverse kinematics consistency by reducing the kinematic error.

\section{Material and methods}

\subsection{Experimental data}

Optoelectronic and biplanar radiographic data was collected from several studies, all of which received ethical approvals (Comite de Protection des Personnes, 2006A00386-45, 2015-A01760-49, 2018-A00173-52). Twenty-six subjects (four females and twenty-two males, age: $24.3 \pm 11.1$ years old, height: $176.2 \pm 7.6 \mathrm{~cm}$, weight: $67.3 \pm 9.4 \mathrm{~kg}, \quad$ BMI: $21.6 \pm 2.14 \mathrm{~m} / \mathrm{kg}^{2}$ ) were equipped with a set of 30 reflective markers: 14 were placed on anatomical landmarks of the lower limbs and adapted from ISB recommendations (Wu et al. 2002); four technical clusters composed of four plate-mounted markers were strapped on both shanks and thighs (Figure 1). These clusters were only used in a solidification procedure to reconstruct incomplete trajectories of hidden markers (Söderkvist and Wedin 1993). They were not used for inverse kinematics since it has been shown to have a minor influence on computed joint angles (Kainz et al. 2016). 


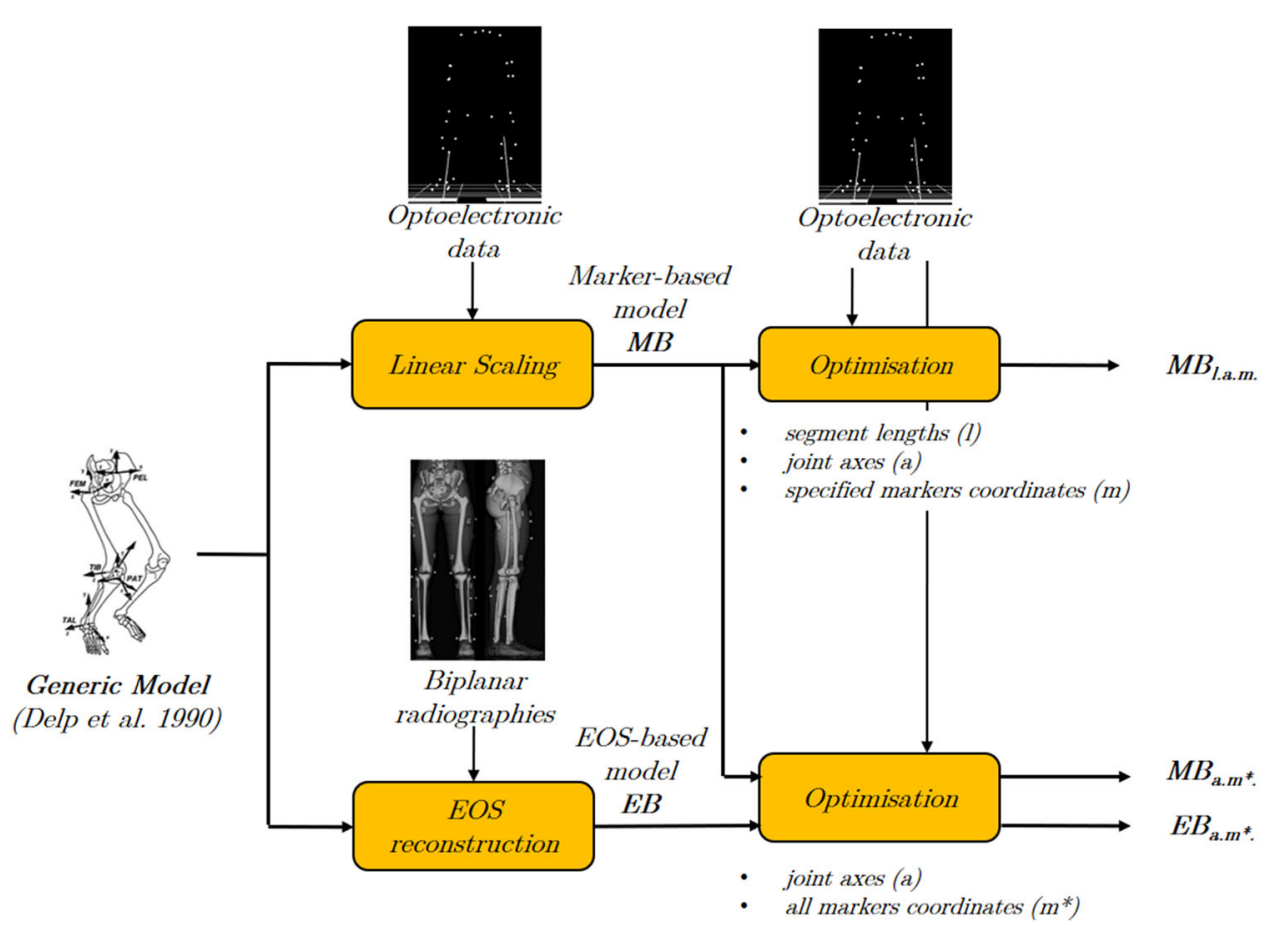

Figure 2. Summary of scaling steps applied to each model. The reader can refer to sections 2.2.2, 2.2.3 and 2.2.4 for more details.

Subjects equipped with markers underwent $\operatorname{EOS}^{\circledR}$ biplanar radiographs (EOS ${ }^{\circledR}$ Imaging, Paris, France), allowing three-dimensional reconstruction of the pelvis, femurs, tibiae and fibulae following a validated protocol (Chaibi et al. 2012) - see Figure 3. This data was used to compute the reference models (imagebased models) presented in section 2.2.3. Hip- and knee-joint functional movements in upright position were recorded for the right and left legs by a 12-cameras motion capture system (Vicon ${ }^{\mathrm{TM}}$ system; Nexus 2 software; Oxford Metrics, UK) at $100 \mathrm{~Hz}$ : flexion/ extension of the knee; flexion/extension, abduction/ adduction and internal and external rotation of the hip (Camomilla et al. 2006). Among the 26 subjects, 6 subjects additionally performed two gait cycles. Markers trajectories were smoothed with a five-frame central window moving average filter. Two passes were done in reverse direction to minimise the shifting effect. Gaps in trajectories were filled using a C2spline interpolation (gaps shorter than 15 frames, i.e., $0.15 \mathrm{~s}$ ) or using a solidification procedure based on the other markers of the same body segment (gaps longer than 15 frames).

\subsection{Geometrical scaling}

For each subject, five geometrical scaling methods issued from the literature were applied on a generic model resulting in five scaled models: two imagebased models (raw and optimised) that were considered as a reference, one linear models and two optimised models. Figure 2 provides acronyms and a sum up of the scaling methods applied. Linear and optimised models were calibrated using algorithms implemented in CusToM (Muller et al. 2019b), an open-source Matlab toolbox dedicated to inverse dynamics based musculoskeletal simulation.

\subsubsection{Generic model}

A predefined 14 Degrees of Freedom (DoF) lower limbs kinematic model was used as a generic model for motion capture data and EOS data. It is adapted from a leg musculoskeletal model available in the AnyBody Managed Model Repository (Delp et al. 1990; Lund et al. 2018) and implemented in CusToM. The lower limbs model had 6 DoF between the ground frame and the pelvis reference frame, 3 rotational DoF at the hip joint centres (HJCs), and 1 rotational DoF (pure hinge joint) at the knee joint centres (KJCs) as in (Reinbolt et al. 2005; Andersen et al. 2010b). The kinematic model was composed of 5 segments (pelvis, both femurs and both shanks), with no foot - ending at the ankle joint centres (AJCs). ISB conventions ( $\mathrm{Wu}$ et al. 2002) were followed for segment frame orientations. The Z-axis of the thigh was used as the knee joint axis. In this model, scalable geometrical parameters were the segment lengths, the orientations of joint axes, and the model marker positions. Depending on the type of scaling method 

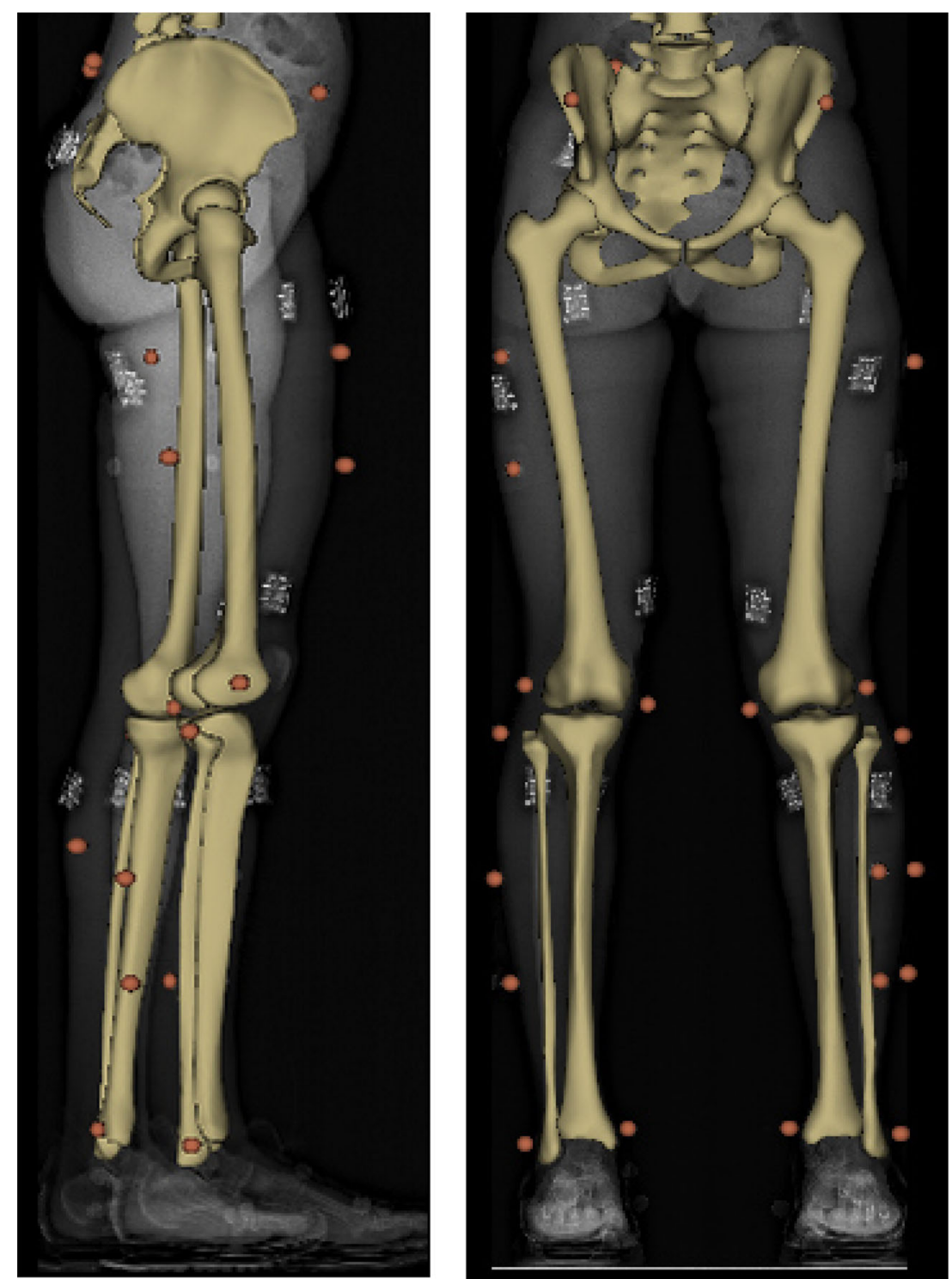

Figure 3. X-rays of a subject equipped with 30 markers and with four four-marker plates. X-rays were acquired with EOS. Reconstruction of bones (pelvis, femurs, tibiae and fibulae) is also shown. The subject is positioned in a 'shifted-feet' standing position to simplify bony structures recognition. This standing position has been previously validated(Chaibi et al. 2012).

applied thereafter, all part of these parameters were scaled.

\subsubsection{Linear scaling method}

One linear-scaled models were computed from the generic model called marker-based linear scaling. Homothetic factors were computed from the ratio between the experimental lengths and the model lengths. The experimental segment lengths were estimated from experimental markers and the generic model segment lengths were estimated from model markers. Pelvis experimental and model lengths were computed with the right and left posterior superior iliac spines (RPSIS, LPSIS) and the right and left anterior superior iliac spines (RASIS, LASIS). Four lengths were computed: RASIS-to-LASIS, RPSIS-toLPSIS, RASIS-to-RPSIS and LASIS-to-LPSIS. The mean of the fourth ratios between experimental and model lengths was used as the homothetic factor of the pelvis. Experimental lengths of the femurs were computed from HJCs (Harrington et al. 2007) and the middle of the medial and lateral epicondyles markers. For the shank, experimental markers placed on knee epicondyles and malleoli were used to estimate knee and ankle joint positions. It resulted in a Marker-Based model (MB) presented in figure 2.

\subsubsection{Image-based scaling method}

An image-based scaling method was applied with EOS data. An EOS-based model (EB) was built and 
Table 1. Markers local coordinates allowed to be optimised (Wu et al. 2002) with $\mathrm{MB}_{\text {l.a.m. }}$ scaling. The seven markers are placed on the right and left sides. $\mathrm{x}, \mathrm{y}, \mathrm{z}$ coordinates are respectively antero-posterior, longitudinal and medio-lateral. With $\mathrm{MB}_{a . m *}$. and $\mathrm{EB}_{a . m *}$. every coordinates are optimised. Marker clusters on thighs and shanks were not considered in geometrical scaling and inverse kinematics. The choice of the coordinates to optimise was based on the analysis of the biomechanical model ensuring a proper convergence of the algorithm.

\begin{tabular}{lcccc}
\hline Palpated landmarks for marker placement & Body segment & optimised x-coordinate & optimised y-coordinate & optimised z-coordinate \\
\hline Posterior superior iliac spine & Pelvis & Yes & No & Yes \\
Anterior superior iliac spine & Pelvis & Yes & No & Yes \\
Lateral femur epicondyle & Thigh & No & No & No \\
Medial femur epicondyle & Thigh & Yes & No & Yes \\
Fibula head & Shank & Yes & No & Yes \\
Lateral malleolus & Shank & No & No & No \\
Medial malleolus & Shank & Yes & No & No \\
\hline
\end{tabular}

considered as a reference for each subject (Melhem et al. 2016). The 3D reconstructions of the lower limb bones were extracted from the biplanar radiographs. Femurs, tibiae and fibulae were reconstructed based on a parametric model and on statistical inferences (Chaibi et al. 2012). Regions were then automatically segmented on the bones. The HJCs were identified with a least-squares sphere fitting on the femoral heads regions of the $3 \mathrm{D}$ mesh (Pillet et al. 2014). Spheres were least-squares fitted on medial and lateral posterior aspects of femoral condyles. The KJCs were defined as the mid-points between the two spheres centres and the knee joint axis by the line passing through them (Sauret et al. 2016). Finally, malleoli regions of the fibulae and the tibiae were selected on the parametric bone models. Their barycentres were used to create middle points which were considered as the AJCs.

Moreover, EOS enabled to place the markers in the segment frame directly from the reconstruction. Markers' locations were computed by adjusting manually $14 \mathrm{~mm}$ diameter sphere models on the biplanar radiographs to match markers contours. The reproductibility of this procedure was previously determined at $0.35 \mathrm{~mm}$ (Sauret et al. 2016). HJCs locations were expressed in the pelvic coordinate system based on the external markers of the pelvis seen on the radiographs ( $\mathrm{Wu}$ et al. 2002). Markers positions placed on femurs and tibiae were expressed in segments frames, following ISB recommendations.

\subsubsection{Optimisation-based scaling methods}

To obtain the three last models of the study, two kinds of optimisation-based scaling methods were applied. Firstly, the optimisation scaling method consisted in optimising homothetic factors of segments, knee joint axes orientations and model markers local coordinates (Table 1). It resulted in the computation of the optimised marker-based model denoted as $\mathrm{MB}_{\text {l.a.m., }}$ with optimised parameters denoted as subscripts: segment lengths ( $l$ ), joint axes $(a)$ and model markers $(m)$.

Secondly, another optimisation-based scaling method was applied on MB and EB models. It consisted in only optimising joint axes orientations and every model markers local coordinates specified in Table 1. It resulted in the computation of the optimised marker-based model denoted as $\mathrm{MB}_{a \cdot m^{*}}$. and $\mathrm{EB}_{a . m^{*}}$. with optimised parameters denoted as subscripts: joint axes $(a)$ and every model markers local coordinates $\left(m^{*}\right)$.

For all methods, hip- and knee-joint functional movements recorded as presented in section 2.1 were used as an input. According to Lund et al. (2015), dynamic trials are relevant for geometrical scaling, especially to study motions involving large joint amplitudes.

The $\mathrm{MB}_{\text {l.a.m. }}$. method was performed using the $\mathrm{MB}$ model as initial guess (see section 2.2.2). The $\mathrm{MB}_{a \cdot m^{*}}$. and $\mathrm{EB}_{a \cdot m^{*}}$. methods used the $\mathrm{MB}$ and $\mathrm{EB}$ models as initial guesses respectively (see Section 2.2.3).

The optimisation-based scaling methods consisted in the following steps (Muller et al. 2015a): $N_{f}$ frames equally spaced in time were extracted from hip- and knee-joint functional movements data. It was set to $N_{f}=100$ after prior experiments as a good trade-off between convergence and computation time. A first inverse kinematics step (Lu and O'Connor 1999) was performed over the selected frames (with MB or EB models) using an interior-point algorithm to get an initial guess of joint angles $\mathbf{q}$. Then, a parameter optimisation step was performed to identify homothetic factors $\mathbf{k}$, rotations $\boldsymbol{\alpha}$ of joint axes and variations of marker local coordinates $\Delta \mathbf{p}$. All variables were normalised between [-1,1] (Reinbolt et al. 2005). These parameters were optimised by minimising the cumulative kinematic error (CKE), denoted as $\Phi$, in Equation 1. The CKE is the cumulative quadratic sum of the Euclidean distances between experimental markers positions $\mathbf{X}_{\text {exp }, m}$ and model markers positions $\mathbf{X}_{\text {mod, } m}^{R_{\text {global }}}$ over the $N_{f}$ selected frames: 


$$
\Phi=\sum_{f}^{N_{f}} \sum_{m}^{N_{m}}\left\|\mathbf{X}_{\text {exp }, m}\left(t_{f}\right)-\mathbf{X}_{\text {mod }, m}^{R_{\text {global }}}\left(\mathbf{q}\left(t_{f}\right), \mathbf{k}, \boldsymbol{\alpha}, \Delta \mathbf{p}\right)\right\|^{2}
$$

where $N_{m}$ is the number of markers in the model. The minimisation was bounded with constraints as shown in Equation (2):

$$
\begin{array}{ll}
\min _{\mathbf{k}, \boldsymbol{\alpha}, \Delta \mathbf{p}} & \Phi\left(\mathbf{q}\left(t_{f}\right), \mathbf{k}, \boldsymbol{\alpha}, \Delta \mathbf{p}\right) \\
\text { s.t. } & \forall s \in\left[\left[1 ; N_{s}\right]\right], \quad\left|\frac{k_{s}}{k_{s}^{0}}-1\right|<20 \% \\
& \forall a \in\left[\left[1 ; N_{\alpha}\right]\right], \quad \alpha_{a, \min }<\alpha_{a}<\alpha_{a, \max } \\
& \forall l c \in\left[\left[1 ; N_{l c}\right]\right], \quad\left|\Delta p_{l c}\right|<0.05 m
\end{array}
$$

where $N_{s}$ is the number of solids, $N_{\alpha}$ is the number of parameters associated to the rotation of joint axes and $N_{l c}$ is the number optimised marker local coordinates. Markers local coordinates to be optimised are adapted from (Andersen et al. 2010b) and ensured the equation system to be determined for a single frame. Homothetic factors were constrained to vary by $\pm 20 \%$ in the $\mathrm{MB}_{\text {l.a.m. }}$. scaling and they did not vary in $\mathrm{MB}_{a . m^{*}}$. and $\mathrm{EB}_{a . m^{*}}$. Marker local coordinates variations were constrained to vary by $\pm 5 \mathrm{~cm}$. Knee joint axes (Z-oriented) were optimised with a X-Y rotation sequence in the thigh local frame. Rotations were constrained to $\pm 20^{\circ}$ around $\mathrm{X}$-axis and to $\pm 30^{\circ}$ around $\mathrm{Y}$-axis.

Finally, inverse kinematics and parameters optimisation were successively executed in a loop until the mean variation of CKE between $(i-1)^{\text {th }}$ and $i^{\text {th }}$ loops was less than $1 \%$.

\subsection{Model evaluation \& statistical analysis}

Models were evaluated thanks to three quantities: geometrical parameters (segment lengths, rotations of joint axes, markers local coordinates), kinematic errors and joint angles.

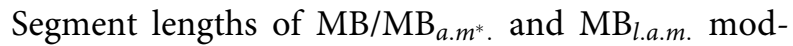
els were compared to the $\mathrm{EB} / \mathrm{EB}_{a . m^{*}}$. reference models and to the literature (Bartels et al. 2015; Kainz et al. 2017): namely inter-hip distance (right to left HJC), femur lengths (HJC to KJC) and shank lengths (KJC to AJC). A non-parametric Friedman's test verified if the segment lengths of the five models among subjects were significantly different $(p<0.05)$. A post-hoc Tukey's HSD test assessed significant differences among models compared to EB models. The $\mathrm{X}$ and $\mathrm{Y}$ rotations of joint axes and variations of markers local coordinates were quantified on optimisation-based models $\mathrm{MB}_{\text {l.a.m. }}, \mathrm{MB}_{a . m^{*} \text {. and }} \mathrm{EB}_{a . m^{*} .}$.
Joint angles of hip- and knee-joint functional movements and gait cycles were computed using inverse kinematics, also called multibody kinematic optimisation ( $\mathrm{Lu}$ and O'Connor 1999; Begon et al. 2017), with markers mentioned in Table 1. They had a weight factor of 1 . Resulting kinematic errors of functional movements were compared for the five models. As for the segment lengths, the same statistical tests were applied for kinematic errors. For gait cycles, joint angles of hips and knees obtained with all models were compared to $\mathrm{EB}_{a . m^{*}}$. results, considered as a reference. One-way ANOVA tests using statistical parametric mapping (SPM) (Pataky et al. 2015) over the duration of the gait trials were computed to compare each model condition as a repeated measure. In case of significant difference, two-tailed t-tests $(p<0.05)$ between models and the reference $\mathrm{EB}_{a \cdot m^{*}}$. were applied to identify when these differences occurred during the gait cycle.

\section{Results}

\subsection{Geometrical parameters}

Segment lengths are presented in Table 2. Distances with respect to the EB model are presented in Figure 4. Significant differences were found among models for each length $(p<0.05$ and $p<0.001)$. Indeed, for the right femur, the mean distances between $\mathrm{MB}$ and $\mathrm{MB}_{\text {l.a.m. }}$. models with the $\mathrm{EB}$ model were respectively, $4.0 \pm 13.8 \mathrm{~mm}$ and $8.4 \pm 12.5 \mathrm{~mm}$. For the left femur, $\mathrm{MB}$ and $\mathrm{MB}_{\text {l.a.m. }}$. models exhibited mean distances of $0.88 \pm 16.3 \mathrm{~mm}$ and $5.1 \pm 13.3 \mathrm{~mm}$.

Mean distance of inter-hip distance with the EB model ranged between $-10.8 \pm 19.9 \mathrm{~mm}(\mathrm{MB})$ and $4.0 \pm 18.5 \mathrm{~mm}\left(\mathrm{MB}_{\text {l.a.m. }}\right)$. Right and left shanks lengths exhibited errors similar to the femurs. Tukey's HSD revealed that right femur and tibia lengths from $\mathrm{MB}_{\text {l.a.m. }}$ models were significantly different from the $\mathrm{EB}$ model. However, inter-hip distance from $\mathrm{MB}$ / $\mathrm{MB}_{a . m^{*}}$. and $\mathrm{MB}_{\text {l.a.m. }}$. were not significantly different from the EB model.

Initial medio-lateral knee axes orientations were optimised in three models: $\mathrm{MB}_{\text {l.a.m. }}, \mathrm{MB}_{a . m^{*}}$. and $\mathrm{EB}_{a . m^{*}}$. Mean and standard deviation associated to successive $\mathrm{X}$ and $\mathrm{Y}$ rotations are presented in Table 3. Both orientation adjustments were about $-2^{\circ}$ to $2^{\circ}$ Standard deviations of $\mathrm{MB}_{\text {l.a.m. }}$ models were higher than other models $\left(3.22^{\circ}\right.$ to $\left.4.06^{\circ}\right)$.

In average, variations of markers local coordinates of $\mathrm{MB}_{\text {l.a.m. }}, \mathrm{MB}_{a . m^{*},}, \mathrm{~EB}_{a . m^{*}}$ models were respectively of $5.6 \pm 12.7 \mathrm{~mm}, 12.3 \pm 9.8 \mathrm{~mm}, 6.3 \pm 7.8 \mathrm{~mm}$. 
Table 2. Segment lengths (mean and standard deviation) for five models: $M B, M B_{l . a . m .}, M B_{a . m^{*} .} E B$ and $E B_{a . m^{*}}$. Friedman's test investigated significant differences between models and Tukey's HSD investigated significant differences with the EB model. ${ }^{*},{ }^{* *},{ }^{* * *}$ indicated respective $p$-values $<0.05,<0.005,<0.001$.

\begin{tabular}{|c|c|c|c|c|c|c|c|}
\hline \multirow[b]{2}{*}{ Right Femur } & \multirow{2}{*}{$\frac{\text { Friedmans' test significance }}{*}$} & \multicolumn{2}{|c|}{$\mathrm{MB} / \mathrm{MB}_{a . m^{*}}$. } & \multicolumn{2}{|c|}{$\mathrm{MB}_{\text {I.a.m. }}$} & \multicolumn{2}{|c|}{$\mathrm{EB} / \mathrm{EB}_{a \cdot m^{*}}$} \\
\hline & & 435.8 & (26.8) & $440.2^{* *}$ & (23.3) & 431.8 & $(23.3)$ \\
\hline Left Femur & $*$ & 434.0 & (27.3) & $438.2^{*}$ & (23.5) & 433.1 & $(22.7)$ \\
\hline Inter-Hip & $* * *$ & 162.7 & (19.8) & 177.4 & (18.4) & 173.5 & $(9.7)$ \\
\hline Right Shank & $* * *$ & 419.6 & (24.8) & $431.5^{* * *}$ & (24.3) & 415.7 & $(23.1)$ \\
\hline Left Shank & $* * *$ & 418.5 & $(25.2)$ & $431.1^{* * *}$ & (24.1) & 414.7 & $(22.7)$ \\
\hline
\end{tabular}

\subsection{Kinematic errors}

Overall, inverse kinematics on hip- and knee-joint functional movements resulted in different kinematic errors (Figure 5). MB models exhibited the largest kinematic errors $(27.4 \pm 3.7 \mathrm{~mm}) . \mathrm{MB}_{\text {l.a.m. }}$ and $\mathrm{MB}_{a . m^{*}}$. models showed lower kinematic errors $(11.0 \pm 1.6 \mathrm{~mm}$ and $6.5 \pm 1.2 \mathrm{~mm}$ ). EB model showed higher kinematic errors than optimised models $(8.9 \pm 3.1 \mathrm{~mm})$ but $\mathrm{EB}_{a . m^{*}}$. models showed the lowest kinematic error $(5.4 \pm 0.8 \mathrm{~mm})$.

Friedman's test revealed significant differences between the models. The kinematic errors of $\mathrm{MB}$ models were significantly higher than these of

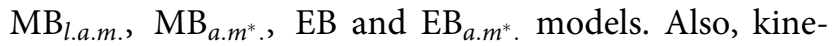
matic errors from $\mathrm{EB}_{a \cdot m^{*}}$. models were significantly lower than $\mathrm{MB}_{\text {l.a.m. }}$ and $\mathrm{EB}$ models. However, no significant difference was found for kinematic errors from $\mathrm{MB}_{a \cdot m^{*}}$. models with respect to $\mathrm{EB}$ and $\mathrm{EB}_{a \cdot m^{*}}$. models. Finally, $\mathrm{MB}_{a \cdot m^{*}}$, $\mathrm{EB}$ and $\mathrm{EB}_{a \cdot m^{*}}$. models had similar kinematic errors.

\subsection{Joint angles}

Among the six subjects who performed two gait cycles each, significant differences in joint angles were identified between models, particularly at the hip joint (Figure 6). Right and left hip flexion angles computed with $\mathrm{EB}_{a . m^{*}}$. model were significantly different from these resulting from the $\mathrm{MB}, \mathrm{MB}_{\text {l.a.m. }}$ and $\mathrm{EB}$ models. Also EB models were significantly different from $\mathrm{EB}_{a . m^{*}}$. models for right and left knee flexion. Differences between angles arose at different instants of the stride, depending on the considered joint.

\section{Discussion}

The primary assumption of the study was that optimisation-based scaling methods should provide the best trade-off between geometrical parameters accuracy with respect to image-based methods and ensure the best inverse kinematics consistency by reducing the kinematic error. To investigate this assumption, 5 scaling methods were applied to a lower limb model on 26 subjects with a
14-DoF generic kinematic model of the lower limbs. The EOS reconstructions of the cohort, considered as the image-based ground truth (Melhem et al. 2016), were a valuable opportunity to assess geometrical scaling methods based on optoelectronic data. The EOS-based models $\left(\mathrm{EB}\right.$ and $\mathrm{EB}_{a . m^{*}}$.) have been considered as a reference for the geometrical parameters and only $\mathrm{EB}_{a \cdot m^{*}}$. models were considered for the joint angles.

\subsection{Geometrical parameters}

Optimising lengths and markers concomitantly induce overfitting for geometrical parameters. Indeed, left and right femur lengths were best evaluated by the markerbased (MB) method which does not optimise segment lengths. However, several studies have claimed that optimising all geometrical parameters is better at estimating segment lengths (Reinbolt et al. 2005; Andersen et al. 2010b; Lund et al. 2015). They reported up to 80, 67.1 , and $10 \mathrm{~mm}$ in segment lengths variations between optimisation-based and linear scaling approaches - see Table 4. However, none of these studies validated the optimised segment lengths directly.

The current study showed significantly lower differences between MB scaled femur lengths and the EB reference compared to the literature (Bartels et al. 2015; Kainz et al. 2017), which exhibited up to $100 \mathrm{~mm}$ differences with CT-scan results. It can be explained by the cohorts of the these studies which were partly pathological (osteoarthritis and cerebral palsy). In the current study, the 26 subjects were healthy and practiced regular physical activity.

Optimisation of joint axes was required because such anatomical aspects differ for every subject (Eckhoff et al. 2003). It led to changes in the orientations of knee joint axes below $5^{\circ}$. These low changes may indicate that the kinematic errors were mostly due to inadequate lengths.

Optimisation of model markers local coordinates was supposed to limit the effects of STA and of the uncertainty of marker placement, even if this last source of error was mitigated by the training of the experimenters (Della Croce et al. 2005). In the last paragraph of the section 3.1, the changes in marker 

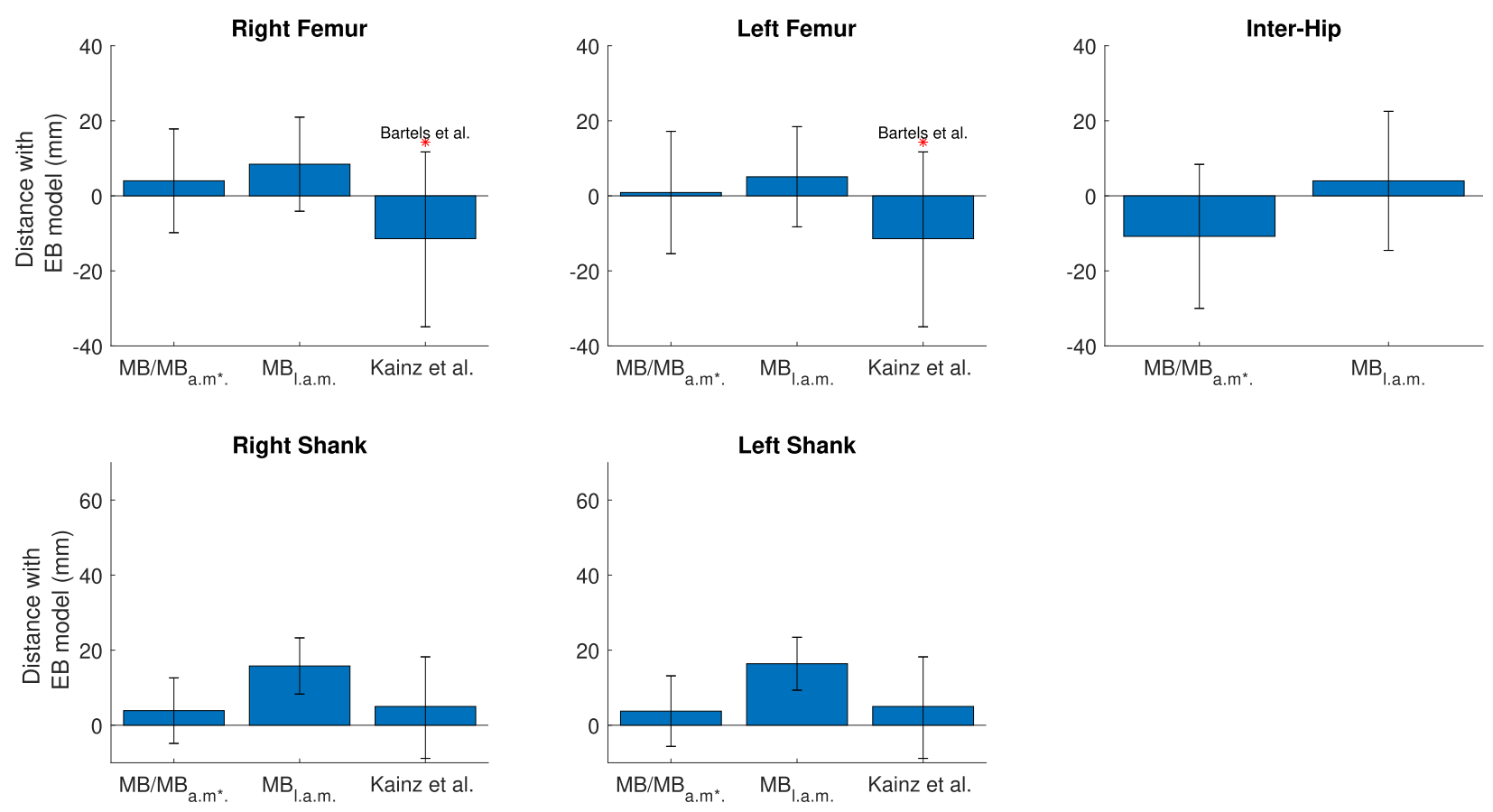

Figure 4. Distance (mean and standard deviation) between segment lengths (femurs, shanks, inter-hip distances) issued from the

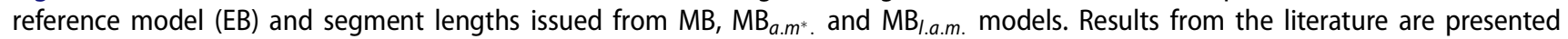
(Bartels et al. 2015; Kainz et al. 2017). Results from Kainz et al. (2017) refer to Figure 1 of the article, in the accuracy column, typically developed participants.

Table 3. Frontal plane rotations $X$ and transverse plane rotations $Y$ (mean and standard deviation) of right and left knee rotation axes for three models: $\mathrm{MB}_{\text {l.a.m. }}, \mathrm{MB}_{a . m^{*}, \mathrm{H}} \mathrm{EB}_{a . m^{*}}$.

\begin{tabular}{|c|c|c|c|c|c|c|c|c|c|c|c|c|}
\hline \multirow{2}{*}{$\begin{array}{l}\text { Optimisation-based models } \\
\text { Rotations }\left({ }^{\circ}\right)\end{array}$} & \multicolumn{4}{|c|}{$\mathrm{MB}_{a \cdot m^{*}}}$. & \multicolumn{4}{|c|}{$\mathrm{MB}_{\text {l.a.m. }}$} & \multicolumn{4}{|c|}{$\mathrm{EB}_{a \cdot m^{*}}}$. \\
\hline & $x$ & & $y$ & & $x$ & & $y$ & & $x$ & & $y$ & \\
\hline Right Knee Axis & 0.44 & (2.26) & -2.06 & (2.68) & 1.21 & (3.59) & -0.54 & $(4.06)$ & 1.94 & $(1.83)$ & -2.02 & (2.1) \\
\hline Left Knee Axis & -0.54 & (2.14) & 1.85 & (2.6) & -1.84 & (3.22) & 0.87 & (3.57) & -0.81 & (1.84) & 0.81 & (2.16) \\
\hline
\end{tabular}

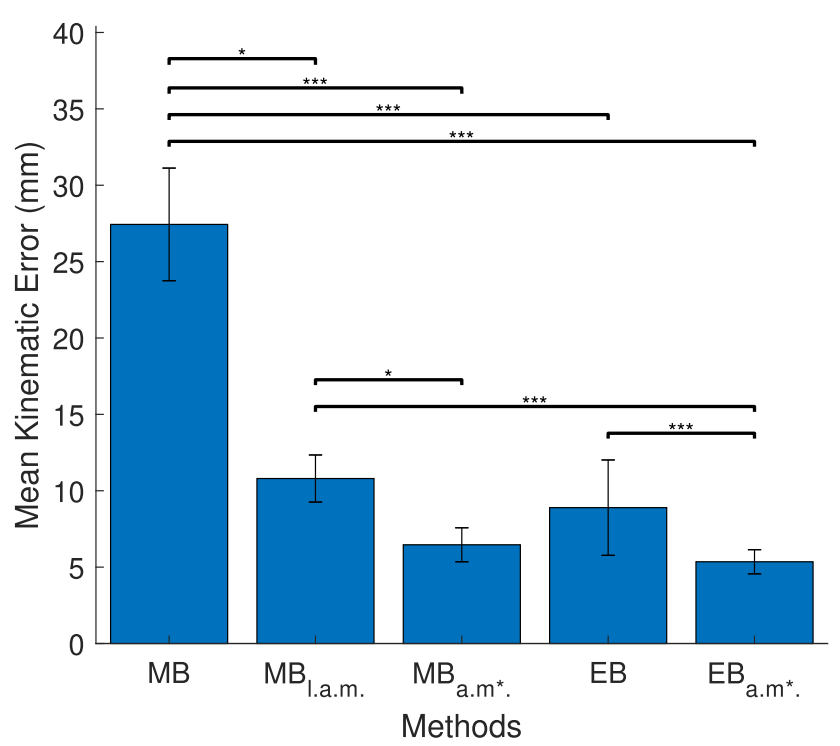

Figure 5. Kinematic errors (mean and standard deviation) on hip- and knee-joint functional movements with five models: $\mathrm{HB}, \mathrm{MB}, \mathrm{MB}_{\text {l.a.m. }}, \mathrm{MB}_{a . m^{*},}, \mathrm{~EB}$ and $\mathrm{EB}_{a . m^{*} .}{ }^{*},{ }^{* *}$, ${ }^{* * *}$ indicated respective $p$-values $<0.05,<0.005,<0.001$ with respect to Tukey's honest significant difference criterion. local coordinates after optimisation were consistent with respect to the literature because they had the same order of magnitude as the reported STA: $20 \mathrm{~mm}$ for the shank, $30 \mathrm{~mm}$ for the thigh and $9 \mathrm{~mm}$ for the pelvis (Leardini et al. 2005; Camomilla et al. 2017). The choice of dynamic trials as an input of the optimisation procedures had an impact on the results, since they are supposed to be subject to STA through the body motion. Indeed, static trials would have given relevant results close to the static configuration but may have suffered from larger kinematics errors for bigger joint angle magnitudes of the motions to be analysed. Therefore, in this study, dynamic trials were preferred to static trials since the motions to be evaluated were gaits, involving large joint amplitudes.

\subsection{Kinematic errors}

Resulting kinematic errors of $\mathrm{MB}_{\text {l.a.m. }} \mathrm{MB}_{a . m^{*} \text {., }} \mathrm{EB}$ and $\mathrm{EB}_{a . m^{*}}$. models were in accordance with literature results of optimisation-based approaches (Reinbolt 

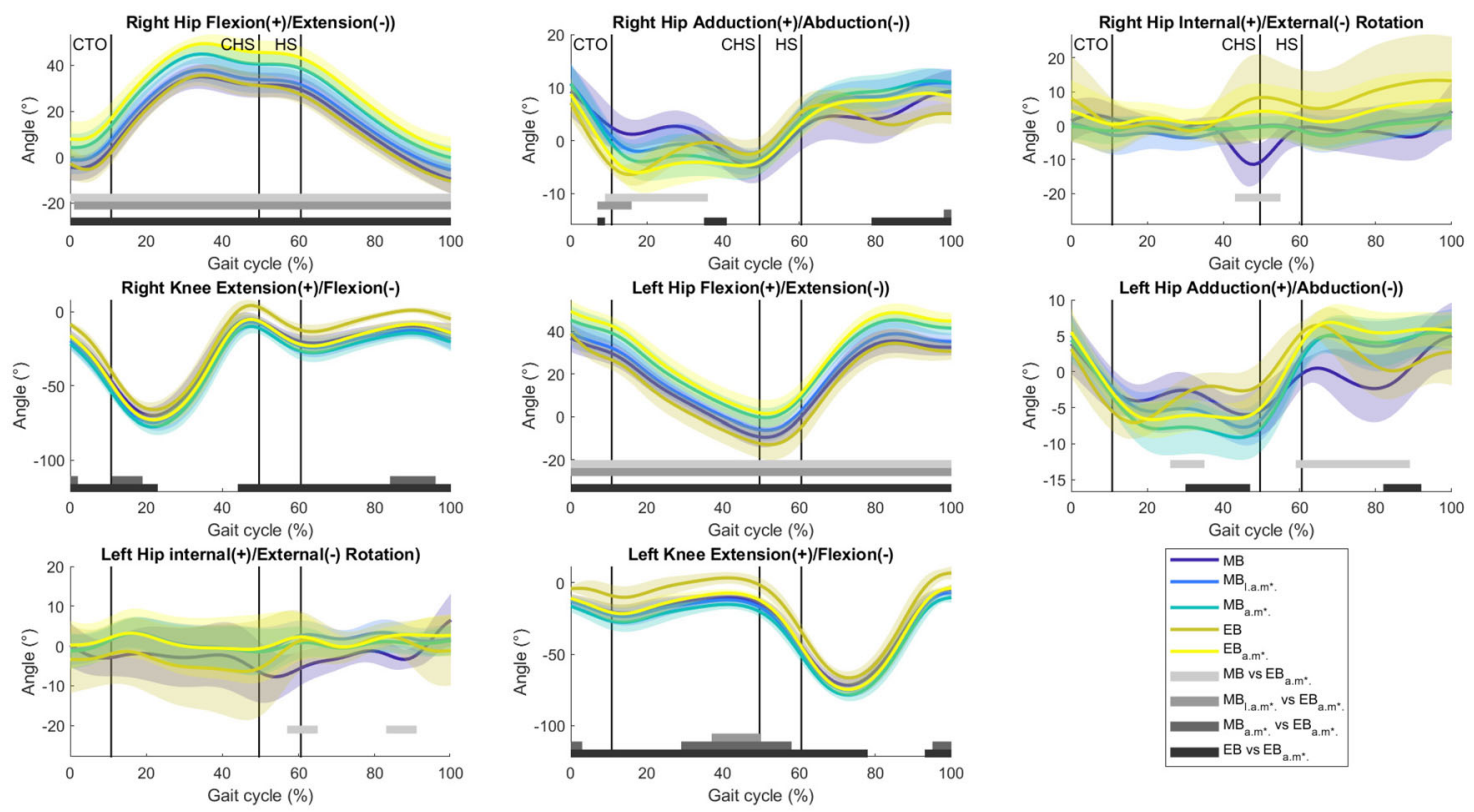

Figure 6. Hip, knee joint angle means over 12 gait trials performed by 6 subjects with a standard deviation cloud for each of the 5 models for one stride. Dark blue line represents the $\mathrm{MB}$ model. Light blue and green lines represent $\mathrm{MB}_{\text {l.a.m., }} \mathrm{MB}_{a . m^{*}}$. models respectively. The EB and $\mathrm{EB}_{a . m^{*}}$. models are in orange and yellow. Shaded rectangles along the $\mathrm{x}$-axis show incidences of significant differences between at least 2 model conditions $(p<0.05)$ after one-way ANOVA and SPM-Bonferroni correction. Vertical bars represent the timing of the gait events. CTO: Contra-lateral toe-off. CHS: Contra-lateral heel-strike. TO: Toe-off.

Table 4. Maximal length variations between scaling methods in the literature.

\begin{tabular}{lcccc}
\hline Maximal length variations reported & Reinbolt et al. 2005 & Andersen et al. 2010b & Lund et al. 2015 & Present study \\
\hline $\begin{array}{l}\text { Value }(\mathrm{mm}) \\
\text { Geometrical parameter }\end{array}$ & 67.1 & 10 & 80 & 14.7 \\
& Right hip location & Left foot length & Right hip location & inter-hip distances \\
\hline
\end{tabular}

et al. 2005, 2007; Andersen et al. 2010b; Lund et al. 2015; Muller et al. 2015a) - see Table 5. However, MB models exhibited larger kinematic errors than the literature. So, tuning only segment lengths is not enough to scale the model, mainly because of misplacements of experimental markers and STA.

On the contrary, the $\mathrm{MB}_{\text {l.a.m. }}$ and $\mathrm{MB}_{a . m^{*}}$. methods were robust to the minimisation of kinematic errors over the 26 subjects, despite various sources of errors (Begon et al. 2017): measurement errors, segment lengths, DoF of the model. However, $\mathrm{MB}_{\text {l.a.m. }}$ model misestimated segment lengths compared to $\mathrm{MB}$ and $\mathrm{MB}_{a \cdot m^{*}}$. models which were closer to the $\mathrm{EB}_{a \cdot m^{*}}$. reference models.

Therefore, the most appropriate method to be applied in marker-based geometrical scaling is an optimisation of marker local coordinates which enables a better location of markers and minimises STA effects. These results are in accordance with the Opensim scaling method (Reinbolt et al. 2005).

\subsection{Joint angles}

The $\mathrm{MB}_{a \cdot m^{*}}$. and $\mathrm{EB}_{a \cdot m^{*}}$. methods exhibited similar joint angles. This is of interest since in these models,
Table 5. Mean kinematic errors of scaled models after inverse kinematics.

\begin{tabular}{lc}
\hline Study/methods & Mean kinematic errors $(\mathrm{mm})$ \\
\hline Reinbolt et al. (2005) & 7.8 \\
Andersen et al. (2010b) & 5.5 \\
Muller et al. (2015a) & 8.6 \\
Lund et al. (2015) & 4.5 \\
Present study - $\mathrm{MB}_{\text {I.a.m. }}$ & 10.8 \\
Present study - $\mathrm{MB}_{a . m^{*} .}$ & 6.5 \\
Present study - EB & 8.8 \\
Present study - $\mathrm{EB}_{a . m^{*} .}$ & 5.3 \\
\hline
\end{tabular}

the segments lengths were very similar and supposed to be accurate. It indicates that the optimisation of the marker local coordinates resulted in a similar effect on the joint angles computation for both methods, whereas $\mathrm{MB}_{\text {l.a.m. }}$ led to different results. Low kinematic errors of $\mathrm{MB}_{a \cdot m^{*}}$. and $\mathrm{EB}_{a \cdot m^{*}}$. does not necessarily mean that joint angles were accurate or that STA were fully compensated (Bonnet et al. 2017). Kinematic accuracy can only really be assessed with intra-cortical pins (Andersen et al. 2010a) or biplanar fluoroscopic data (Gasparutto et al. 2015; Richard et al. 2017). However, the consistency between both results and the low kinematic error suggests that the primary assumption of the study, i.e., that 
optimisation-based scaling methods should provide the best trade-off between geometrical parameters accuracy with respect to image-based methods and ensure the best inverse kinematics consistency, is not supported. It seems more relevant to use $\mathrm{MB}_{a \cdot m^{*}}$. instead of $\mathrm{MB}_{\text {l.a.m. }}$. to scale the model in a musculoskeletal study.

\subsection{Methodological limitations}

The study has several limitations that should be noted. First of all, optimisation-based methods may lead to overfitting. It tends to spread the experimental and model errors on the optimised geometrical parameters, namely the segment lengths, the marker local coordinates and the joint axes. To ensure a good balance between correct segment lengths and low kinematic errors, well-chosen constraints and correct initial guesses are required. The optimisation-based methods are robust and guarantee to reduce kinematic errors but the results of the study show that it is preferable to exclude the segment lengths of the lower limbs from any optimisation-based scaling.

Computationally, a subject was scaled in less than 4 minutes for $N_{f}=100$ frames $(3.10 \mathrm{GHz}$ laptop, 32 Go RAM). This computation time may be reduced by working on the choice and the number of frames to include in the optimisation (Muller et al. 2015b). The study was also limited by the way the scaling was defined: the homothetic factor was uniformly applied in all directions of a given segment. It has consequences on the scaling of large bones such as the pelvis. A solution may be to declare multiple scaling factors for each segment and optimise them separately (Rassmussen et al. 2005; Delp et al. 2007) with wellchosen markers weights in the optimisation (Trinler and Baker 2018). Non-linear functions could also be used to enhance the scaling properties before an optimisation as in $\mathrm{MB}_{a \cdot m^{*}}$, as it has been done in Lund et al. (2015); Zhang et al. (2016); Nolte et al. (2019). Another enhancement may be to use a database of models extracted from MRIs and to get a closer initial guess, as proposed in (Ding et al. 2019; Klemt et al. 2019).

Feet scaling is another issue that was not handled in the current study. Feet data from the EOS biplanar images have to be considered for a future extended protocol.

Last, the knee model was limited to one rotational DoF and its validity is still discussed in the literature (Gasparutto et al. 2015; Clément et al. 2015): according to Kainz et al. 2016, knee models have an influence on computed joint angles. In the future, more complex models could be evaluated to confirm the use of $\mathrm{MB}_{a \cdot m^{*}}$. for scaling.

\section{Conclusion}

The current study aimed at identifying a scaling method based on optoelectronic data offering the best trade-off between accurate geometrical parameters (segment lengths, orientation of joint axes, marker coordinates) and consistent inverse kinematics outputs (joint angles and kinematic error). The primary assumption of the study was that optimisation-based scaling methods should provide the best trade-off between geometrical parameters accuracy with respect to image-based methods and ensure the best inverse kinematics consistency. It has not been supported by the results, since the optimised segment lengths obtained with $\mathrm{MB}_{\text {l.a.m. }}$. were significantly different from the these obtained from the EOS reference measures, and led to an higher kinematic error. Finally, the marker-based scaling with optimised model marker coordinates and joint axes in $\mathrm{MB}_{a \cdot m^{*}}$. gave the most consistent scaling and joint angles with regard to these obtained from the EOS measures. This study is of interest since this scaling is the first step to be applied to a model in any musculoskeletal study. An interesting future work may be to investigate the effect of such scaling on the muscle paths and the moment arms of the model that are fundamental to run accurate musculoskeletal analyses.

\section{Disclosure statement}

No potential conflict of interest was reported by the author(s).

\section{Funding}

The present study was partly funded by the chair of the enhanced soldier in the digital battlefield from the StCyr Foundation.

\section{ORCID}

P. Puchaud (D) http://orcid.org/0000-0002-9335-630X

C. Sauret (D) http://orcid.org/0000-0002-6196-2698

A. Muller (D) http://orcid.org/0000-0003-2606-1429

N. Bideau (D) http://orcid.org/0000-0001-5333-7991

G. Dumont (iD http://orcid.org/0000-0002-0709-0921

H. Pillet (D) http://orcid.org/0000-0002-0263-5910

C. Pontonnier (D) http://orcid.org/0000-0003-1140-3772 


\section{References}

Andersen MS, Benoit DL, Damsgaard M, Ramsey DK, Rasmussen J. 2010. Do kinematic models reduce the effects of soft tissue artefacts in skin marker-based motion analysis? An in vivo study of knee kinematics. J Biomech. 43(2):268-273.

Andersen MS, Damsgaard M, MacWilliams B, Rasmussen J. 2010. A computationally efficient optimisation-based method for parameter identification of kinematically determinate and over-determinate biomechanical systems. Comput Methods Biomech Biomed Eng. 13(2): 171-183.

Bartels W, Demol J, Gelaude F, Jonkers I, Vander Sloten J. 2015. Computed tomography-based joint locations affect calculation of joint moments during gait when compared to scaling approaches. Comput Methods Biomech Biomed Eng. 18(11):1238-1251.

Begon M, Andersen MS, Dumas R. 2017. Multibody kinematic optimization for the estimation of upper and lower limb human joint kinematics: a systematic review. J Biomech Eng. 140(3):1-11.

Blemker SS, Asakawa DS, Gold GE, Delp SL. 2007. Imagebased musculoskeletal modeling: Applications, advances, and future opportunities. J Magn Reson Imag. 25(2): 441-451.

Bonnet V, Dumas R, Cappozzo A, Joukov V, Daune G, Kulić D, Fraisse P, Andary S, Venture G. 2017. A constrained extended Kalman filter for the optimal estimate of kinematics and kinetics of a sagittal symmetric exercise. J Biomech. 62:140-147.

Camomilla V, Bonci T, Cappozzo A. 2017. Soft tissue displacement over pelvic anatomical landmarks during 3-D hip movements. J Biomech. 62:14-20.

Camomilla V, Cereatti A, Vannozzi G, Cappozzo A. 2006. An optimized protocol for hip joint centre determination using the functional method. J Biomech. 39(6): 1096-1106.

Chaibi Y, Cresson T, Aubert B, Hausselle J, Neyret P, Hauger O, de Guise JA, Skalli W. 2012. Fast 3D reconstruction of the lower limb using a parametric model and statistical inferences and clinical measurements calculation from biplanar X-rays. Comput Methods Biomech Biomed Eng. 15(5):457-466.

Clément J, Dumas R, Hagemeister N, de Guise JA. 2015. Soft tissue artifact compensation in knee kinematics by multi-body optimization: Performance of subject-specific knee joint models. J Biomech. 48(14):3796-3802.

Damsgaard M, Rasmussen J, Christensen ST, Surma E, de Zee M. 2006. Analysis of musculoskeletal systems in the AnyBody Modeling System. Simul Model Pract Theory. 14(8):1100-1111.

Della Croce U, Leardini A, Chiari L, Cappozzo A. 2005. Human movement analysis using stereophotogrammetry: Part 4: assessment of anatomical landmark misplacement and its effects on joint kinematics. Gait \& Posture. 21(2): 226-237.

Delp SL, Anderson FC, Arnold AS, Loan P, Habib A, John CT, Guendelman E, Thelen DG. 2007. OpenSim: OpenSource Software to Create and Analyze Dynamic Simulations of Movement. IEEE Trans Biomed Eng. 54(11):1940-1950.
Delp SL, Loan JP, Hoy MG, Zajac FE, Topp EL, Rosen JM. 1990. An Interactive Graphics-Based Model of the Lower Extremity to Study Orthopaedic Surgical Procedures. IEEE Trans Biomed Eng. 37(8):757-767.

Ding Z, Tsang CK, Nolte D, Kedgley AE, Bull AM. 2019. Improving musculoskeletal model scaling using an anatomical atlas: the importance of gender and anthropometric similarity to quantify joint reaction forces. IEEE Trans Biomed Eng. 66(12):3444-3456.

Dupré T, Dietzsch M, Komnik I, Potthast W, David S. 2018. Agreement of measured and calculated muscle activity during highly dynamic movements modelled with a spherical knee joint. J Biomech. 84:73-80.

Eckhoff DG, Bach JM, Spitzer VM, Reinig KD, Bagur MM, Baldini TH, Rubinstein D, Humphries S. 2003. Threedimensional morphology and kinematics of the distal part of the femur viewed in virtual reality: Part II. J Bone Joint Surg - Ser A. 85(supp. 4):97-104.

Gasparutto X, Sancisi N, Jacquelin E, Parenti-Castelli V, Dumas R. 2015. Validation of a multi-body optimization with knee kinematic models including ligament constraints. J Biomech. 48(6):1141-1146.

Halonen KS, Dzialo CM, Mannisi M, Venäläinen MS, De Zee M, Andersen MS. 2017. Workflow assessing the effect of gait alterations on stresses in the medial tibial cartilage - Combined musculoskeletal modelling and finite element analysis. Sci Rep. 7(1):17396.

Hamner SR, Delp SL. 2013. Muscle contributions to foreaft and vertical body mass center accelerations over a range of running speeds. J Biomech. 46(4):780-787.

Handsfield GG, Meyer CH, Hart JM, Abel MF, Blemker SS. 2014. Relationships of 35 lower limb muscles to height and body mass quantified using MRI. J Biomech. 47(3): 631-638.

Harrington ME, Zavatsky AB, Lawson SEM, Yuan Z, Theologis TN. 2007. Prediction of the hip joint centre in adults, children, and patients with cerebral palsy based on magnetic resonance imaging. J Biomech. 40(3): 595-602.

Kainz H, Hoang HX, Stockton C, Boyd RR, Lloyd DG, Carty CP. 2017. Accuracy and reliability of marker-based approaches to scale the pelvis, thigh, and shank segments in musculoskeletal models. J Appl Biomech. 33(5): 354-360.

Kainz H, Modenese L, Lloyd DG, Maine S, Walsh HP, Carty CP. 2016. Joint kinematic calculation based on clinical direct kinematic versus inverse kinematic gait models. J Biomech. 49(9):1658-1669. Available from: https://www.sciencedirect. com/science/article/abs/pii/S0021929016304134. doi:10. 1016/j.jbiomech.2016.03.052.

Klemt C, Nolte D, Ding Z, Rane L, Quest RA, Finnegan ME, Walker M, Reilly P, Bull AM. 2019. Anthropometric Scaling of Anatomical Datasets for Subject-Specific Musculoskeletal Modelling of the Shoulder. Ann Biomed Eng. 47(4):924-936.

Leardini A, Chiari A, Della Croce U, Cappozzo A. 2005. Human movement analysis using stereophotogrammetry Part 3. Soft tissue artifact assessment and compensation. Gait Posture. 21(2):212-225.

Lu TW, O’Connor JJ. 1999. Bone position estimation from skin marker co-ordinates using global optimisation with joint constraints. J Biomech. 32(2):129-134. 
Lund ME, Andersen MS, de Zee M, Rasmussen J. 2015. Scaling of musculoskeletal models from static and dynamic trials. Int Biomech. 2(1):1-11.

Lund ME, Damsgaard M, Tørholm S, Galibarov PE, Jung M. 2018. The AnyBody Managed Model Repository (AMMR) (Version 2.1.1)

Marra MA, Vanheule V, Fluit R, Koopman B, Rasmussen J, Verdonschot N, Andersen MS. 2015. A Subject-Specific Musculoskeletal Modeling Framework to Predict In Vivo Mechanics of Total Knee Arthroplasty. J Biomech Eng. 137(2):020904

Melhem E, Assi A, El Rachkidi R, Ghanem I. 2016. EOS® biplanar X-ray imaging: concept, developments, benefits, and limitations. J Child Orthop. 10(1):1-14.

Muller A, Germain C, Pontonnier C, Dumont G. 2015a. A Simple Method to Calibrate Kinematical Invariants: Application to Overhead Throwing. Int Soc Biomech Sports. (2007):2-5.

Muller A, Pontonnier C, Dumont G. 2019a. Motion-based prediction of hands and feet contact efforts during asymmetric handling tasks. IEEE Trans Biomed Eng. doi: 10.1109/TBME.2019.2913308.

Muller A, Pontonnier C, Germain C, Dumont G. 2015. Dealing with modularity of multibody models. Comput Methods Biomech Biomed Eng. 18(sup1):2008-2009. (November):

Muller A, Pontonnier C, Puchaud P, Dumont G. 2019. CusToM: a Matlab toolbox for musculoskeletal simulation. JOSS. 4(33):927-924.

Nolte D, Bull AMJ, Kedgley AE. 2019. Bone shape reconstruction from MoCap data with soft tissue corrections using statistical shape models. 25th Congress of the European Society of Biomechanics; Vienna, Austria. p. 1.

Pataky T, Robinson M, Vanrenterghem J. 2015. Corrigendum to Vector field statistical analysis of kinematic and force trajectories. J Biomech. 48(1):190-192.

Pillet H, Sangeux M, Hausselle J, El Rachkidi R, Skalli W. 2014. A reference method for the evaluation of femoral head joint center location technique based on external markers. Gait Posture. 39(1):655-658.

Rassmussen J, de Zee M, Damsgaard M, Christensen ST, Clemens M, Siebertz K. 2005. A General Method for Scaling Musculo-Skeletal Models. In: International Symposium on Computer Simulation in Biomechanics; Cleveland, $\mathrm{OH}$, United States.

Reinbolt JA, Haftka RT, Chmielewski TL, Fregly BJ. 2007. Are patient-specific joint and inertial parameters necessary for accurate inverse dynamics analyses of gait?. IEEE Trans Biomed Eng. 54(5):782-793.
Reinbolt JA, Schutte JF, Fregly BJ, Koh BI, Haftka RT, George AD, Mitchell KH. 2005. Determination of patient-specific multi-joint kinematic models through two-level optimization. J Biomech. 38(3):621-626.

Richard V, Cappozzo A, Dumas R. 2017. Comparative assessment of knee joint models used in multi-body kinematics optimisation for soft tissue artefact compensation. J Biomech. 62:95-101.

Sauret C, Pillet H, Skalli W, Sangeux M. 2016. On the use of knee functional calibration to determine the mediolateral axis of the femur in gait analysis: Comparison with EOS biplanar radiographs as reference. Gait Posture. 50:180-184.

Scheys L, Jonkers I, Loeckx D, Maes F, Spaepen A, Suetens P. 2006. Image based musculoskeletal modeling allows personalized biomechanical analysis of gait. In: Biomedical simulation ISBMS 2006. Lecture Notes in Computer Science; vol. 4072. Berlin: Springer. p. 58-66.

Scheys L, Jonkers I, Schutyser F, Pans S, Spaepen A, Suetens P. 2005. Image based methods to generate subject-specific musculoskeletal models for gait analysis. Int Cong Ser. 1281:62-67.

Söderkvist I, Wedin PÅ. 1993. Determining the movements of the skeleton using well-configured markers. J Biomech. 26(12):1473-1477.

Trinler U, Baker R. 2018. Estimated landmark calibration of biomechanical models for inverse kinematics. Med Eng Phys. 51:79-83.

Valente G, Pitto L, Testi D, Seth A, Delp SL, Stagni R, Viceconti M, Taddei F. 2014. Are subject-specific musculoskeletal models robust to the uncertainties in parameter identification?. PLoS ONE. 9(11):e112625.

van den Bogert AJ, Geijtenbeek T, Even-Zohar O, Steenbrink F, Hardin EC. 2013. A real-time system for biomechanical analysis of human movement and muscle function. Med Biol Eng Comput. 51(10):1069-1077.

van den Bogert AJ, Smith GD, Nigg BM. 1994. In vivo determination of the anatomical axes of the ankle joint complex: An optimization approach. J Biomech. 27(12): 1477-1488.

Wu G, Siegler S, Allard P, Kirtley C, Leardini A, Rosenbaum D, Whittle M, D'Lima DD, Cristofolini L, Witte $\mathrm{H}$, et al. 2002. ISB recommendation on definitions of joint coordinate system of various joints for the reporting of human joint motionâpart I: ankle, hip, and spine. J Biomech. 35(4):543-548.

Zhang J, Fernandez J, Hislop-Jambrich J, Besier TF. 2016. Lower limb estimation from sparse landmarks using an articulated shape model. J Biomech. 49(16):3875-3881. 